Research Article

\title{
Derivative-Based Trapezoid Rule for the Riemann-Stieltjes Integral
}

\author{
Weijing Zhao and Zhaoning Zhang \\ College of Air Traffic Management, Civil Aviation University of China, Tianjin 300300, China \\ Correspondence should be addressed to Weijing Zhao; 693509394@qq.com
}

Received 23 January 2014; Revised 14 April 2014; Accepted 14 April 2014; Published 30 April 2014

Academic Editor: Ker-Wei Yu

Copyright ( 2014 W. Zhao and Z. Zhang. This is an open access article distributed under the Creative Commons Attribution License, which permits unrestricted use, distribution, and reproduction in any medium, provided the original work is properly cited.

The derivative-based trapezoid rule for the Riemann-Stieltjes integral is presented which uses 2 derivative values at the endpoints. This kind of quadrature rule obtains an increase of two orders of precision over the trapezoid rule for the Riemann-Stieltjes integral and the error term is investigated. At last, the rationality of the generalization of derivative-based trapezoid rule for RiemannStieltjes integral is demonstrated.

\section{Introduction}

In mathematics, the Riemann-Stieltjes integral is a kind of generalization of the Riemann integral, named after Bernhard Riemann and Thomas Stieltjes. It is Stieltjes that first gave the definition of this integral [1] in 1894. It serves as an instructive and useful precursor of the Lebesgue integral. It is known that the Riemann-Stieltjes integral has wide applications in the field of probability theory [2,3], stochastic process [4], and functional analysis [5], especially in original formulation of F. Riesz's theorem and the spectral theorem for self-adjoint operators in a Hilbert space.

Definite integration is one of the most important and basic concepts in mathematics. And it has numerous applications in fields such as physics and engineering. In several practical problems, we need to calculate integrals. As is known to all, as for $I=\int_{a}^{b} f(x) d x$, once the primitive function $F(x)$ of integrand $f(x)$ is known, the definite integral of $f(x)$ over the interval $[a, b]$ is given by NewtonLeibniz formula, that is,

$$
\int_{a}^{b} f(x) d x=F(b)-F(a) .
$$

However, the explicit primitive function $F(x)$ is not available or its primitive function is not easy to obtain, such as $e^{ \pm x^{2}}, \sin x^{2}$, and $(\sin x) / x$. Moreover, the integrand $f(x)$ is only available at certain points $x_{i}, i=1,2, \ldots, n$. How to get high-precision numerical integration formulas becomes one of the challenges in fields of mathematics [6].

The methods of quadrature are usually based on the interpolation polynomials and can be written in the following form:

$$
\int_{a}^{b} f(x) d x \approx \sum_{i=0}^{n} w_{i} f\left(x_{i}\right)
$$

where there are $n+1$ distinct integration points at $x_{0}, x_{1}, \ldots, x_{n}$ within the interval $[a, b]$ and $n+1$ weights $w_{i}$. If the integration points are uniformly distributed over the interval, so $x_{i}=x_{0}+i h$ in which $h=(b-a) / n$.

These $w_{i}$ can be derived in several different ways [7-9]. One is interpolate $f(x)$ at the $n+1$ points $x_{0}, x_{1}, \ldots, x_{n}$, using the Lagrange polynomials and then integrating the foresaid polynomials to obtain (2).

The other is based on the precision of a quadrature formula. Select the $w_{i}, i=0,1, \ldots, n$, so that the error,

$$
R_{n}(f)=\int_{a}^{b} f(x) d x-\sum_{i=0}^{n} w_{i} f\left(x_{i}\right),
$$

is exactly zero for $f(x)=x^{j}, j=0,1, \ldots, n$. Using the method of undetermined coefficients, this approach generates a system of $n+1$ linear equations for weights $w_{i}$. 
Since the monomials $1, x, \ldots, x^{n}$ are linearly independent, the linear system of equations has a unique solution.

The trapezoidal rule is the most well-known numerical integration rule of this type. Trapezoidal rule for classical Riemann integral is

$$
\int_{a}^{b} f(x) d x=\frac{b-a}{2}(f(a)+f(b))-\frac{(b-a)^{3}}{12} f^{\prime \prime}(\xi)
$$

where $\xi \in(a, b)$.

In spite of the many accurate and efficient methods for numerical integration being available in [7-9], recently Mercer has obtained trapezoid rule for Riemann-Stieltjes integral which engender a generalization of Hadamard's integral inequality [10]. Then he develops Midpoint and Simpson's rules for Riemann-Stieltjes integral [11] by using the concept of relative convexity. Burg has proposed derivativebased closed Newton-Cotes numerical quadrature [12] which uses both the function value and the derivative value on uniformly spaced intervals. Zhao and Li have proposed midpoint derivative-based closed Newton-Cotes quadrature [13] and numerical superiority has been shown. Recently, Simos and his partners have made a contribution to the Newton-Cotes formula for the Riemann integral and its applications [1421], especially the connection between closed Newton-Cotes, trigonometrically fitted differential methods, symplectic integrators, and efficient solution of the Schrodinger equation [17-21].

Motivation for the research presented here lies in construction of derivative-based trapezoid rule for the RiemannStieltjes integral, which is generalization of the results in [10-12]. In this paper, the derivative-based trapezoid rule for the Riemann-Stieltjes integral is presented. This new scheme is investigated in Section 2. In Section 3, the error term is presented. Finally, conclusions are drawn in Section 4.

\section{Derivative-Based Trapezoid Rule for the Riemann-Stieltjes Integral}

In this section, by adding the derivatives at the endpoints, derivative-based trapezoid rule for the Riemann-Stieltjes integral is presented.

Theorem 1. Suppose that $f^{\prime}$ and $g$ are continuous on $[a, b]$ and $g$ is increasing there. The derivative-based trapezoid rule for the Riemann-Stieltjes integral is

$$
\begin{aligned}
\int_{a}^{b} f(t) d g & \\
\approx T= & \left(\frac{6}{(b-a)^{2}} \int_{a}^{b} \int_{a}^{t} g(x) d x d t\right. \\
& -\frac{12}{(b-a)^{3}} \int_{a}^{b} \int_{a}^{t} \int_{a}^{y} g(x) d x d y d t \\
& -g(a)) f(a)
\end{aligned}
$$

$$
\begin{aligned}
& +\left(g(b)-\frac{6}{(b-a)^{2}} \int_{a}^{b} \int_{a}^{t} g(x) d x d t\right. \\
& \left.+\frac{12}{(b-a)^{3}} \int_{a}^{b} \int_{a}^{t} \int_{a}^{y} g(x) d x d y d t\right) f(b) \\
& +\left(\frac{2}{b-a} \int_{a}^{b} \int_{a}^{t} g(x) d x d t\right. \\
& \left.-\frac{6}{(b-a)^{2}} \int_{a}^{b} \int_{a}^{t} \int_{a}^{y} g(x) d x d y d t\right) f^{\prime}(a) \\
& +\left(\frac{4}{b-a} \int_{a}^{b} \int_{a}^{t} g(x) d x d t\right. \\
& -\frac{6}{(b-a)^{2}} \int_{a}^{b} \int_{a}^{t} \int_{a}^{y} g(x) d x d y d t \\
& \left.-\int_{a}^{b} g(t) d t\right) f^{\prime}(b) .
\end{aligned}
$$

Proof. Looking for the derivative-based trapezoid rule for the Riemann-Stieltjes integral, we seek numbers $a_{0}, a_{1}, b_{0}, b_{1}$ such that

$$
\int_{a}^{b} f(t) d g \approx a_{0} f(a)+a_{1} f(b)+b_{0} f^{\prime}(a)+b_{1} f^{\prime}(b)
$$

is equality for $f(t)=1, t, t^{2}, t^{3}$. That is,

$$
\begin{aligned}
& \int_{a}^{b} 1 d g=a_{0}+a_{1} \\
& \int_{a}^{b} t d g=a_{0} a+a_{1} b+b_{0}+b_{1} \\
& \int_{a}^{b} t^{2} d g=a_{0} a^{2}+a_{1} b^{2}+2 b_{0} a+2 b_{1} b \\
& \int_{a}^{b} t^{3} d g=a_{0} a^{3}+a_{1} b^{3}+3 b_{0} a^{2}+3 b_{1} b^{2}
\end{aligned}
$$

Therefore,

$$
\begin{aligned}
& a_{0}+a_{1}=g(b)-g(a) \\
& a_{0} a+a_{1} b+b_{0}+b_{1}=b g(b)-a g(a)-\int_{a}^{b} g(t) d t \\
& a_{0} a^{2}+a_{1} b^{2}+2 b_{0} a+2 b_{1} b \\
& =b^{2} g(b)-a^{2} g(a)-2 b \int_{a}^{b} g(t) d t \\
& \quad+2 \int_{a}^{b} \int_{a}^{t} g(x) d x d t
\end{aligned}
$$




$$
\begin{aligned}
a_{0} a^{3} & +a_{1} b^{3}+3 b_{0} a^{2}+3 b_{1} b^{2} \\
= & b^{3} g(b)-a^{3} g(a)-3 b^{2} \int_{a}^{b} g(t) d t \\
& +6 b \int_{a}^{b} \int_{a}^{t} g(x) d x d t \\
& -6 \int_{a}^{b} \int_{a}^{t} \int_{a}^{y} g(x) d x d y d t
\end{aligned}
$$

Solving simultaneous (8) for $a_{0}, a_{1}, b_{0}, b_{1}$, we obtain

$$
\begin{aligned}
a_{0}= & \frac{6}{(b-a)^{2}} \int_{a}^{b} \int_{a}^{t} g(x) d x d t \\
& -\frac{12}{(b-a)^{2}} \int_{a}^{b} \int_{a}^{t} \int_{a}^{y} g(x) d x d y d t-g(a) ; \\
a_{1}= & g(b)-\frac{6}{(b-a)^{2}} \int_{a}^{b} \int_{a}^{t} g(x) d x d t \\
& +\frac{12}{(b-a)^{2}} \int_{a}^{b} \int_{a}^{t} \int_{a}^{y} g(x) d x d y d t ; \\
b_{0}= & \frac{2}{b-a} \int_{a}^{b} \int_{a}^{t} g(x) d x d t \\
& -\frac{6}{(b-a)^{2}} \int_{a}^{b} \int_{a}^{t} \int_{a}^{y} g(x) d x d y d t ; \\
b_{1}= & \frac{4}{b-a} \int_{a}^{b} \int_{a}^{t} g(x) d x d t \\
& -\frac{6}{(b-a)^{2}} \int_{a}^{b} \int_{a}^{t} \int_{a}^{y} g(x) d x d y d t-\int_{a}^{b} g(t) d t .
\end{aligned}
$$

So we have the derivative-based trapezoid rule for the Riemann-Stieltjes integral as desired.

Corollary 2. The precision of the derivative-based trapezoid rule for the Riemann-Stieltjes integral is 3.

Proof. From the construction of $a_{0}, a_{1}, b_{0}, b_{1}$, we obtain that the derivative-based trapezoidal rule for the RiemannStieltjes integral has degree of precision not less than 3 .

In Section 3 Theorem 3, we can easily see that the quadrature is not equality for $f(t)=t^{4}$. So the precision of this method is 3 .

\section{The Error Term for Riemann-Stieltjes Derivative-Based Trapezoid Rule}

In this section, the error term of the derivative-based trapezoid rule for the Riemann-Stieltjes is investigated. The error term can be found in mainly 3 different ways $[8,9]$.

Here, we use the concept of precision to calculate the error term, where the error term is related to the difference between the quadrature formula for the monomial $x^{p+1} /(p+1)$ ! and the exact value $(1 /(p+1) !) \int_{a}^{b} x^{p+1} d x$, where $p$ is the precision of the quadrature formula.

Theorem 3. Suppose that $f^{(4)}$ and $g^{\prime}$ are continuous on $[a, b]$ and $g$ is increasing there. The derivative-based trapezoid rule for the Riemann-Stieltjes integral with the error term is

$$
\begin{aligned}
& \int_{a}^{b} f(t) d g \\
& =\left(\frac{6}{(b-a)^{2}} \int_{a}^{b} \int_{a}^{t} g(x) d x d t\right. \\
& \quad-\frac{12}{(b-a)^{3}} \int_{a}^{b} \int_{a}^{t} \int_{a}^{y} g(x) d x d y d t \\
& +\left(\frac{g(a))}{g}\right) \frac{6}{(b-a)^{2}} \int_{a}^{b} \int_{a}^{t} g(x) d x d t \\
& \left.+\frac{12}{(b-a)^{3}} \int_{a}^{b} \int_{a}^{t} \int_{a}^{y} g(x) d x d y d t\right) f(b) \\
& +\left(\frac{2}{b-a} \int_{a}^{b} \int_{a}^{t} g(x) d x d t\right. \\
& \left.-\frac{6}{(b-a)^{2}} \int_{a}^{b} \int_{a}^{t} \int_{a}^{y} g(x) d x d y d t\right) f^{\prime}(a)
\end{aligned}
$$

$$
\begin{aligned}
& +\left(\frac{4}{b-a} \int_{a}^{b} \int_{a}^{t} g(x) d x d t\right. \\
& +\frac{6}{(b-a)^{2}} \int_{a}^{b} \int_{a}^{t} \int_{a}^{y} g(x) d x d y d t \\
& \left.\quad-\int_{a}^{b} g(t) d t\right) f^{\prime}(b)
\end{aligned}
$$$$
+\left(\frac{(b-a)^{2}}{12} \int_{a}^{b} \int_{a}^{t} g(x) d x d t\right.
$$

$$
\begin{aligned}
& -\frac{b-a}{12} \int_{a}^{b} \int_{a}^{t} \int_{a}^{y} g(x) d x d y d t \\
& \left.+\int_{a}^{b} \int_{a}^{t} \int_{a}^{z} \int_{a}^{y} g(x) d x d y d z d t\right)
\end{aligned}
$$

$\times f^{(4)}(\xi) g^{\prime}(\eta)$ 
where $\xi, \eta \in(a, b)$. And the error term $R[f]$ of this method is

$$
\begin{aligned}
& \left(\frac{(b-a)^{2}}{12} \int_{a}^{b} \int_{a}^{t} g(x) d x d t\right. \\
& \quad-\frac{b-a}{12} \int_{a}^{b} \int_{a}^{t} \int_{a}^{y} g(x) d x d y d t \\
& \left.\quad+\int_{a}^{b} \int_{a}^{t} \int_{a}^{z} \int_{a}^{y} g(x) d x d y d z d t\right) f^{(4)}(\xi) g^{\prime}(\eta) .
\end{aligned}
$$

Proof. Let $f(t)=t^{4} / 4$ !.

So

$$
\begin{aligned}
\frac{1}{4 !} \int_{a}^{b} t^{4} d g= & \frac{1}{24}\left(b^{4} g(b)-a^{4} g(a)\right) \\
& -\frac{b^{3}}{6} \int_{a}^{b} g(t) d t+\frac{b^{2}}{2} \int_{a}^{b} \int_{a}^{t} g(x) d x \\
& -b \int_{a}^{b} \int_{a}^{t} \int_{a}^{y} g(x) d x d y d t \\
& +\int_{a}^{b} \int_{a}^{t} \int_{a}^{z} \int_{a}^{y} g(x) d x d y d z d t .
\end{aligned}
$$

By Theorem 1, we have

$$
\begin{aligned}
T= & \frac{6}{(b-a)^{2}} \int_{a}^{b} \int_{a}^{t} g(x) d x d t \\
& \left.-\frac{12}{(b-a)^{3}} \int_{a}^{b} \int_{a}^{t} \int_{a}^{y} g(x) d x d y d t-g(a)\right) \frac{a^{4}}{24} \\
+ & \left(g(b)-\frac{6}{(b-a)^{2}} \int_{a}^{b} \int_{a}^{t} g(x) d x d t\right. \\
& \left.+\frac{12}{(b-a)^{3}} \int_{a}^{b} \int_{a}^{t} \int_{a}^{y} g(x) d x d y d t\right) \frac{b^{4}}{24} \\
+ & \left(\frac{2}{b-a} \int_{a}^{b} \int_{a}^{t} g(x) d x d t\right. \\
& \left.-\frac{6}{(b-a)^{2}} \int_{a}^{b} \int_{a}^{t} \int_{a}^{y} g(x) d x d y d t\right) \frac{a^{3}}{6} \\
+ & \left(\frac{4}{b-a} \int_{a}^{b} \int_{a}^{t} g(x) d x d t\right. \\
& \left.-\frac{6}{(b-a)^{2}} \int_{a}^{b} \int_{a}^{t} \int_{a}^{y} g(t) d t\right)^{b} \frac{b^{3}}{6} .
\end{aligned}
$$

By Equations (12)-(13), we obtain

$$
\begin{aligned}
\frac{1}{4 !} \int_{a}^{b} t^{4} d g-T \\
=\frac{(b-a)^{2}}{12} \int_{a}^{b} \int_{a}^{t} g(x) d x d t \\
\quad-\frac{b-a}{12} \int_{a}^{b} \int_{a}^{t} \int_{a}^{y} g(x) d x d y d t \\
\quad+\int_{a}^{b} \int_{a}^{t} \int_{a}^{z} \int_{a}^{y} g(x) d x d y d z d t .
\end{aligned}
$$

This implies that

$$
\begin{aligned}
R[f]= & \frac{(b-a)^{2}}{12} \int_{a}^{b} \int_{a}^{t} g(x) d x d t \\
& -\frac{b-a}{12} \int_{a}^{b} \int_{a}^{t} \int_{a}^{y} g(x) d x d y d t \\
& \left.+\int_{a}^{b} \int_{a}^{t} \int_{a}^{z} \int_{a}^{y} g(x) d x d y d z d t\right) \\
& \times f^{(4)}(\xi) g^{\prime}(\eta) .
\end{aligned}
$$

Corollary 4. Conditions are the same as for Theorem 3. When $g(t)=t$, (10) reduces to the derivative-based trapezoid rule (see [12]) for the classical Riemann integral.

Proof. It is easy to obtain

$$
\begin{aligned}
& \int_{a}^{b} \int_{a}^{t} x d x d t=\frac{1}{6} b^{3}-\frac{1}{2} a^{2} b+\frac{1}{3} a^{3}, \\
& \int_{a}^{b} \int_{a}^{t} \int_{a}^{y} x d x d y d t \\
& \quad=\frac{1}{24} b^{4}-\frac{1}{4} a^{2} b^{2}+\frac{1}{3} a^{3} b-\frac{1}{8} a^{4}, \\
& \int_{a}^{b} \int_{a}^{t} \int_{a}^{z} \int_{a}^{y} x d x d y d z d t \\
& \quad=\frac{1}{120} b^{5}-\frac{1}{12} a^{2} b^{3}+\frac{1}{6} a^{3} b^{2}-\frac{1}{8} a^{3} b+\frac{1}{30} a^{5} .
\end{aligned}
$$

By Theorem 3,

$$
\begin{aligned}
\int_{a}^{b} f(t) d g= & \int_{a}^{b} f(t) d t \\
= & \left(\frac{6}{(b-a)^{2}} \int_{a}^{b} \int_{a}^{t} x d x d t\right. \\
& \left.\quad-\frac{12}{(b-a)^{3}} \int_{a}^{b} \int_{a}^{t} \int_{a}^{y} x d x d y d t-a\right) f(a)
\end{aligned}
$$




$$
\begin{aligned}
& +\left(b-\frac{6}{(b-a)^{2}} \int_{a}^{b} \int_{a}^{t} x d x d t\right. \\
& \left.+\frac{12}{(b-a)^{3}} \int_{a}^{b} \int_{a}^{t} \int_{a}^{y} x d x d y d t\right) f(b) \\
& +\left(\frac{2}{b-a} \int_{a}^{b} \int_{a}^{t} x d x d t\right. \\
& \left.-\frac{6}{(b-a)^{2}} \int_{a}^{b} \int_{a}^{t} \int_{a}^{y} x d x d y d t\right) f^{\prime}(a) \\
& +\left(\frac{4}{b-a} \int_{a}^{b} \int_{a}^{t} x d x d t\right. \\
& -\frac{6}{(b-a)^{2}} \int_{a}^{b} \int_{a}^{t} \int_{a}^{y} x d x d y d t \\
& \left.-\int_{a}^{b} t d t\right) f^{\prime}(b) \\
& +\left(\frac{(b-a)^{2}}{12} \int_{a}^{b} \int_{a}^{t} x d x d t\right. \\
& -\frac{b-a}{12} \int_{a}^{b} \int_{a}^{t} \int_{a}^{y} x d x d y d t \\
& \left.+\int_{a}^{b} \int_{a}^{t} \int_{a}^{z} \int_{a}^{y} x d x d y d z d t\right) f^{(4)}(\xi) \\
& =\frac{b-a}{2}(f(a)+f(b)) \\
& +\frac{(b-a)^{2}}{12}\left(f^{\prime}(a)-f^{\prime}(b)\right) \\
& +\frac{(b-a)^{5}}{720} f^{(4)}(\xi) \text {. }
\end{aligned}
$$

Remark 5. From Corollary 4, we know that the results in Theorem 3 possess the reducibility. When $g(t)=t$, formula (10) reduces to the derivative-based trapezoid rule for the classical Riemann integral. So Theorem 3 is a reasonable generalization of the results in [12].

\section{Conclusions}

We briefly summarize our main conclusions in this paper as follows.

(1) The derivative-based trapezoid rule for the RiemannStieltjes integral is presented which uses 2 derivative values at the endpoints.

(2) This kind of quadrature rule obtains an increase of two orders of precision over the trapezoid rule for the Riemann-Stieltjes integral.
(3) The error term for Riemann-Stieltjes derivative-based trapezoid rule is investigated. And the rationality of the generalization of derivative-based trapezoid rule for Riemann-Stieltjes integral is demonstrated.

The derivative-based midpoint and Simpson's rules for the Riemann-Stieltjes integral will be achieved by further research.

\section{Conflict of Interests}

The authors declare that there is no conflict of interests regarding the publication of this paper.

\section{Acknowledgments}

This work is supported by the Scientific Research Foundation of Civil Aviation University of China (no. 2013QD01X), the Fundamental Research Funds for the Central Universities (no. 3122014C023), and the National Natural Science Foundation of China (no. 71171190).

\section{References}

[1] R. A. Gordon, The Integrals of Lebesgue, Denjoy, Perron, and Henstock, vol. 4 of Graduate Studies in Mathematics, American Mathematical Society, Providence, RI, USA, 1994.

[2] P. Billingsley, Probability and Measure, Wiley Series in Probability and Mathematical Statistics, John Wiley \& Sons, New York, NY, USA, 3rd edition, 1995.

[3] L. Egghe, "Construction of concentration measures for general Lorenz curves using Riemann-Stieltjes integrals," Mathematical and Computer Modelling, vol. 35, no. 9-10, pp. 1149-1163, 2002.

[4] P. E. Kopp, Martingales and Stochastic Integrals, Cambridge University Press, Cambridge, UK, 1984.

[5] W. Rudin, Functional Analysis, International Series in Pure and Applied Mathematics, McGraw-Hill, New York, NY, USA, 2nd edition, 1991.

[6] D. H. Bailey and J. M. Borwein, "High-precision numerical integration: progress and challenges," Journal of Symbolic Computation, vol. 46, no. 7, pp. 741-754, 2011.

[7] K. E. Atkinson, An Introduction to Numerical Analysis, John Wiley \& Sons, New York, NY, USA, 2nd edition, 1989.

[8] R. L. Burden and J. D. Faires, Numerical Analysis, Brooks/Cole, Boston, Mass, USA, 9th edition, 2011.

[9] E. Isaacson and H. B. Keller, Analysis of Numerical Methods, John Wiley \& Sons, New York, NY, USA, 1966.

[10] P. R. Mercer, "Hadamard's inequality and trapezoid rules for the Riemann-Stieltjes integral," Journal of Mathematical Analysis and Applications, vol. 344, no. 2, pp. 921-926, 2008.

[11] P. R. Mercer, "Relative convexity and quadrature rules for the Riemann-Stieltjes integral," Journal of Mathematical Inequalities, vol. 6, no. 1, pp. 65-68, 2012.

[12] C. O. E. Burg, "Derivative-based closed Newton-Cotes numerical quadrature," Applied Mathematics and Computation, vol. 218, no. 13, pp. 7052-7065, 2012.

[13] W. Zhao and H. Li, "Midpoint derivative-based closed NewtonCotes quadrature," Abstract and Applied Analysis, vol. 2013, Article ID 492507, 10 pages, 2013. 
[14] Z. Kalogiratou and T. E. Simos, "Newton-Cotes formulae for long-time integration," Journal of Computational and Applied Mathematics, vol. 158, no. 1, pp. 75-82, 2003.

[15] T. E. Simos, "New closed Newton-Cotes type formulae as multilayer symplectic integrators," Journal of Chemical Physics, vol. 133, no. 10, Article ID 104108, 2010.

[16] T. E. Simos, "New open modified Newton Cotes type formulae as multilayer symplectic integrators," Applied Mathematical Modelling, vol. 37, no. 4, pp. 1983-1991, 2013.

[17] T. E. Simos, "Closed Newton-Cotes trigonometrically-fitted formulae for the solution of the Schrödinger equation," MATCH. Communications in Mathematical and in Computer Chemistry, vol. 60, no. 3, pp. 787-801, 2008.

[18] T. E. Simos, "Closed Newton-Cotes trigonometrically-fitted formulae of high order for the numerical integration of the Schrödinger equation," Journal of Mathematical Chemistry, vol. 44, no. 2, pp. 483-499, 2008.

[19] I. Alolyan and T. E. Simos, "New open modified trigonometrically-fitted Newton-Cotes type multilayer symplectic integrators for the numerical solution of the Schrödinger equation," Journal of Mathematical Chemistry, vol. 50, no. 4, pp. 782-804, 2012.

[20] T. E. Simos, "High order closed Newton-Cotes exponentially and trigonometrically fitted formulae as multilayer symplectic integrators and their application to the radial Schrödinger equation," Journal of Mathematical Chemistry, vol. 50, no. 5, pp. 1224-1261, 2012.

[21] T. E. Simos, "Accurately closed Newton-Cotes trigonometrically-fitted formulae for the numerical solution of the Schrödinger equation," International Journal of Modern Physics C, vol. 24, no. 3, Article ID 1350014, 20 pages, 2013. 


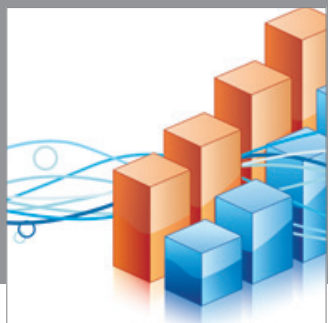

Advances in

Operations Research

mansans

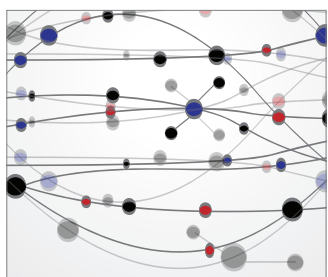

The Scientific World Journal
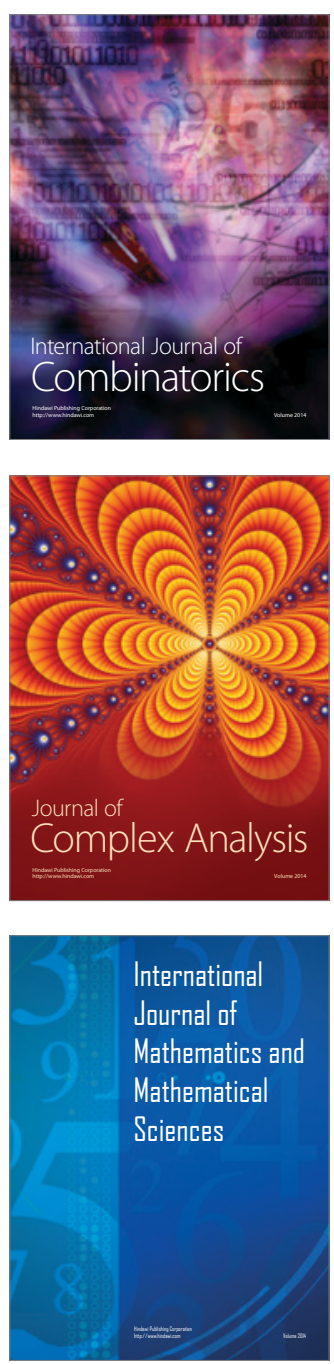
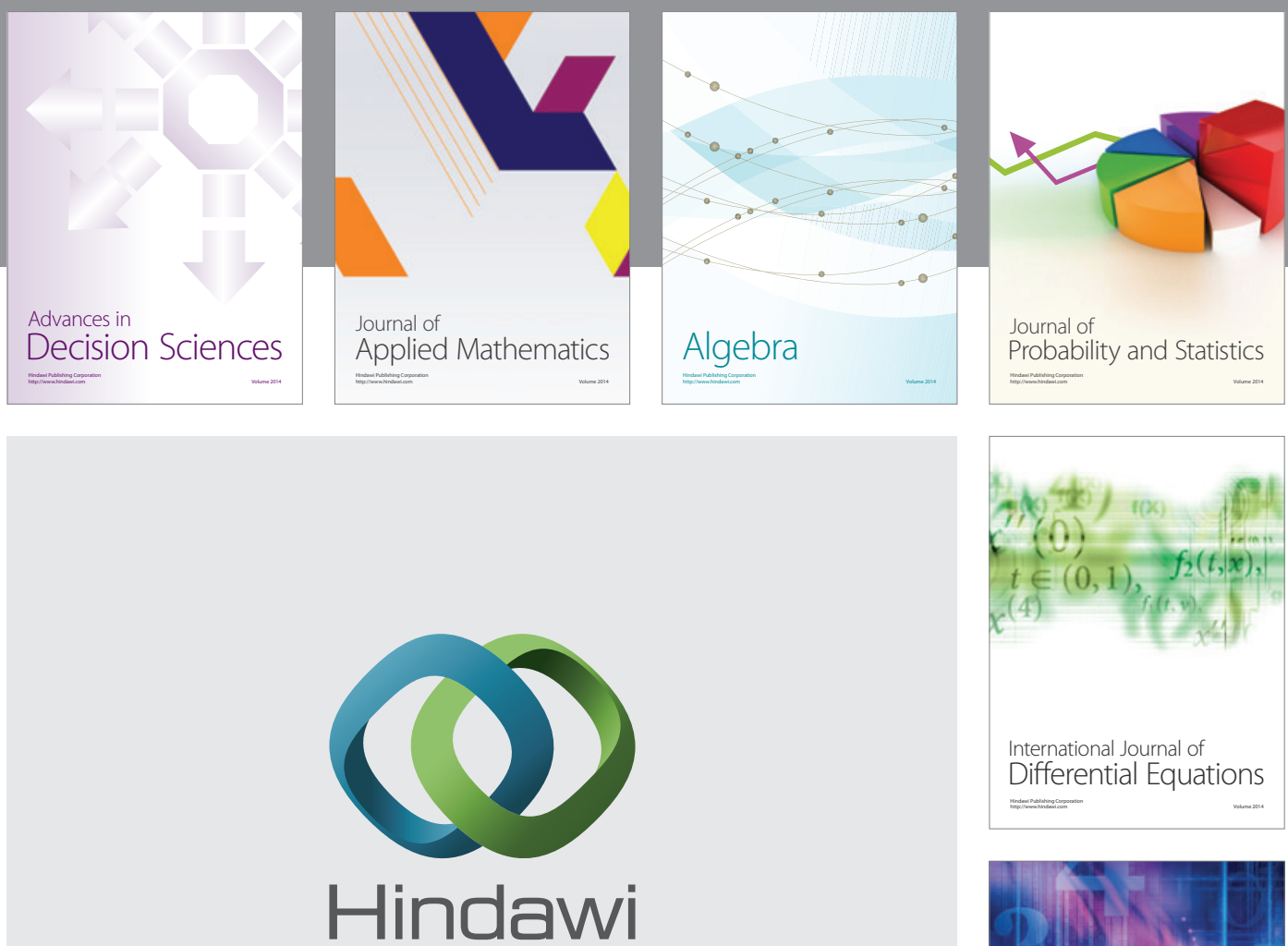

Submit your manuscripts at http://www.hindawi.com
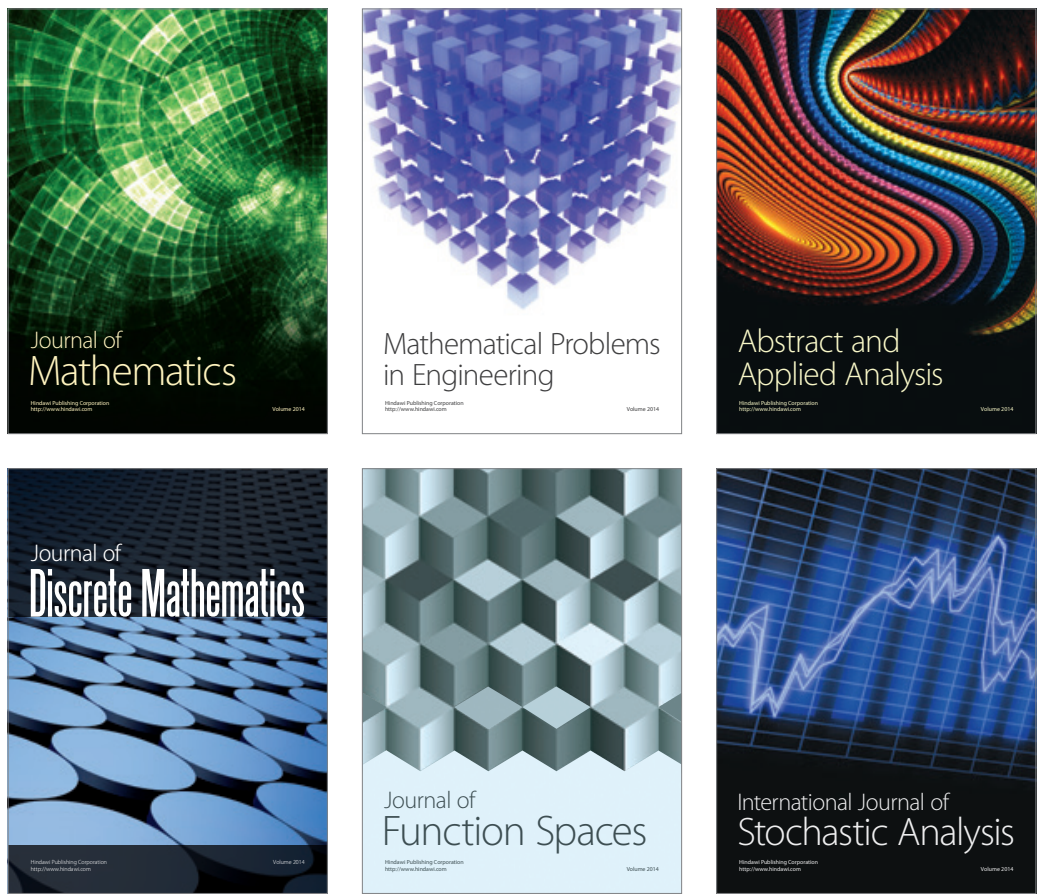

Journal of

Function Spaces

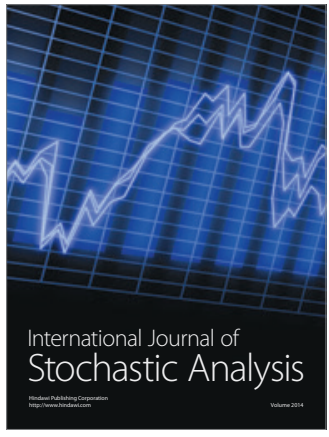

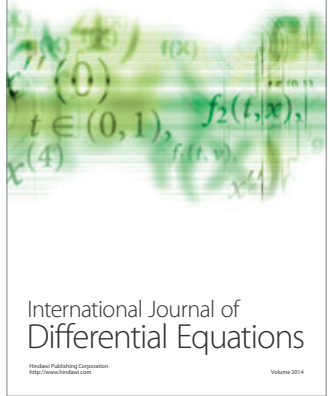
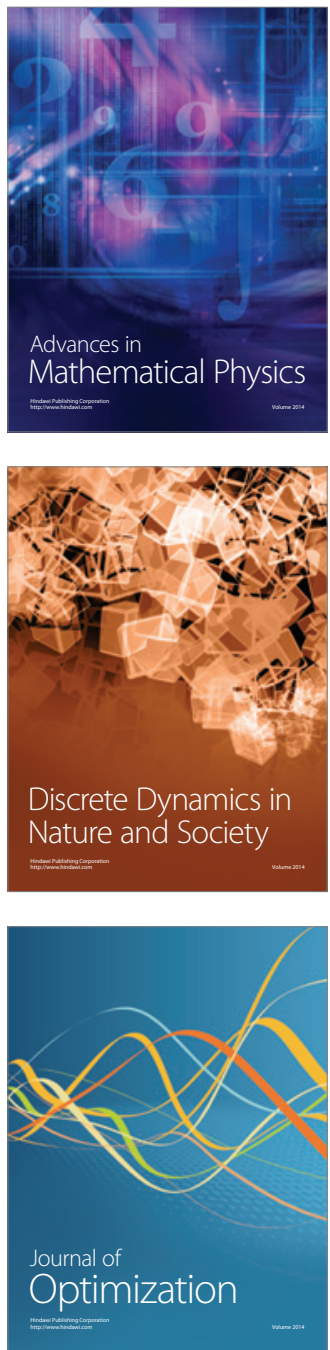